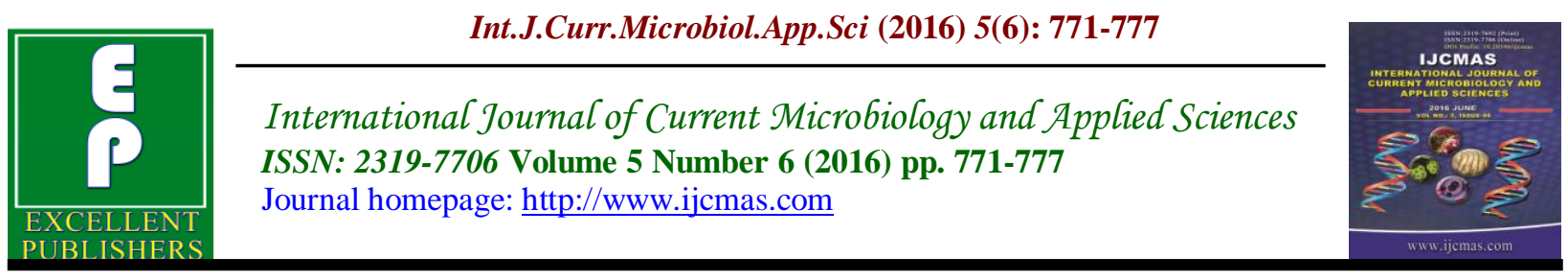

Original Research Article http://dx.doi.org/10.20546/ijcmas.2016.506.085

\title{
Antibiogram of Clinical Isolates of Pseudomonas aeruginosa against Selected Quinolones
}

\author{
Sandhya Mishra*, Mrityunjay Kumar, Anamika Vyas and A.S.Dalal \\ Department of Microbiology, Geetanjali Medical College and Hospital, Udaipur, \\ Rajasthan, India \\ *Corresponding author
}

Keywords

Pseudomonas aeruginosa, Quinolones

\begin{tabular}{l}
\hline Article Info \\
\hline Accepted: \\
25 May 2016 \\
Available Online: \\
10 June 2016
\end{tabular}

A B S T R A C T

One important class of anti-pseudomonal drug is the Fluoroquinolones (FQ) which is also available in oral form, in most countries. However, P. aeruginosa easily becomes resistant to FQs, which severely limits its usefulness. We carried out an antibiogram analysis of five FQs viz. Ciprofloxacin, Ofloxacin, Levofloxacin, Prulifloxacin and Moxifloxacin on $80 P$. aeruginosa clinical isolates. This prospective study was carried out in the Department of Microbiology of a tertiary care teaching hospital in southern Rajasthan. For the same, 80 non-repetitive $P$. aeruginosa isolates, obtained from various clinical samples were assed for resistance to five Fluoroquinolones (FQs) by using Kirby-Bauer disc diffusion method. The resistance patterns were noted and statically analyzed to find any significance. A significant resistance $(\mathrm{p}<0.05)$ was noted towards Ciprofloxacin, Ofloxacin, Levofloxacin in the $P$. aeruginosa isolates obtained from out-patients (OPD) when compared to intensive care unit (ICUs). On the other hand, significant resistance $(\mathrm{p}<0.05)$ for Ofloxacin, Prulifloxacin and Moxifloxacin was obtained for the isolates from wards when compared to the ICU. The overall resistance rates of Ciprofloxacin, Ofloxacin and Levofloxacin were significantly higher than those to Prulifloxacin $(\mathrm{p}=0.000)$. Also Moxifloxacin was significantly resistant when compared to all other FQs tested $(\mathrm{p}=0.000)$. The resistance to FQs was more in the OPDs when compared to the ICUs, while Moxifloxacin had the highest resistance rates. The probable causes for this may relate to previous exposure to FQs.

\section{Introduction}

Fluoroquinolone (FQs) are a novel class of antimicrobial agents; which have a broader acceptance in treating hospitalized and community patients. Introduced in the year 1986, fluoroquinolones are modified form of its precursors-Quinolones. The quinolones act by inhibiting topoisomerase II and DNA gyrase in Gram positive and Gram negative bacteria respectively; thereby interfering with DNA synthesis in the bacteria. Fluoroquinolone are effective for wide range of pathogenic bacteria; as in particular; Ciprofloxacin in infections caused by Pseudomonas aeruginosa. As we approached the fourth decade of FQs usage; its persistence injudicious usage had led to the emergence of FQs resistance; especially in gram negative bacteria such as in 
Pseudomonas aeruginosa (Tanya Strateva et al.,2009; Hideki Kobayashi, 2013)

P. aeruginosa is a Gram negative pathogen with efficient environment adaptability. It is also responsible for a wide range of acute and chronic infections. Its persistence in hospital environment, resistance to several classes of antibiotics and continuous association with serious hospital acquired infections such as sepsis, UTI, etc. presents a therapeutic challenge to the clinicians. P.aeruginosa is also increasingly being acquired in the community. All these factors contribute for $P$. aeruginosa as a common cause of morbidity and mortality. Among the few antimicrobial classes currently available for eradication of $P$. aeruginosa infection which can be used alone or in combination, involves the use of aminoglycosides, $\beta$-lactams, and prominent role is played by FQs. The availability of orally administrable form of $F Q s$ and its minimum toxic effect had increased its convenience of use. (Tanya Strateva et al., 2009; Algun et al., 2004)

FQs have been widely used for the treatment of $P$. aeruginosa infection; however P.aeruginosa is capable of acquiring resistance during antibiotic therapy which is a significant risk factor for the selection of resistant $P$. aeruginosa strains in an individual patient and / or in the populations. Studies have reported a worldwide decline in susceptibility pattern of ciprofloxacin, a most potent congener of FQs. Although some members of the class (Temafloxacin, Trovafloxacin, Grepafloxacin and Gatifloxacin) have been withdrawn or restricted because of adverse events, new members continue to be developed and approved (Prulifloxacin and Moxifloxacin) (Luna Adhikari et al., 2010; Liam Redgrave et al., 2014; Axel Dalhoff, 2012).The present study was designed to evaluate the resistance pattern in $P$. aeruginosa isolates from various clinical samples among different patient population (both in-door and out-door) from our hospital setup against the five FQs (Ciprofloxacin, Ofloxacin, Levofloxacin, Prulifloxacin and Moxifloxacin).

\section{Materials and Methods}

The present prospective study was carried out for one year from 2013-2014 in the department of microbiology of a tertiary care teaching hospital in south Rajasthan. Institutional ethical committee clearance was obtained for the study.

During the study period a total of $80 P$. aeruginosa strains were isolated from various clinical samples and were included in the study. No duplicate strain from the same patient was included in the study. For the culture, clinical samples received were cultured on Nutrient agar, Blood agar and MacConkey agar. Media plates were incubated at a temperature of 37 degrees $\mathrm{C}$ for 18-24 hours. The culture plates were processed using standard microbiological procedure. Characterization and identification was carried out using a combination of colony morphology, Gram stain characteristics, motility tests, pigmentation, oxidation-fermentation test, catalase test, oxidase test and pyocyanin production (Monica Cheesbrough, second edition, part 2).

All the 80 isolates thus obtained; were subjected to antibiotic susceptibility testing on Mueller-Hinton agar plates by the KirbyBauer disc diffusion method as defined by CLSI 2014 guidelines. The panel of FQs to which susceptibility was evaluated consisted of five Fluoroquinolones viz. Ciprofloxacin $(5 \mu \mathrm{g})$, Ofloxacin $(5 \mu \mathrm{g})$, Levofloxacin $(5 \mu \mathrm{g})$, Prulifloxacin $(5 \mu \mathrm{g})$ and Moxifloxacin $(5 \mu \mathrm{g})$. 
The control strain used in this study was P.aeruginosa ATCC 27853.Zone size interpretation was done according to the criteria depicted in table no. I.

The obtained resistance pattern for the above mentioned FQs was subjected to statistical analysis by the ANOVA test and the post hoc Tukey HSD test.

\section{Result and Discussion}

Out of 80 clinical isolates of $P$. aeruginosa; highest contribution of $40 \%$ and $33.75 \%$ were from respiratory secretions and urinary isolates, respectively. Following this, Pus, blood and pleural/billiary fluid contributed $26.25 \%$.

On the basis of patient admission; $76.25 \%$ of the $P$. aeruginosa isolates were from samples obtained from in-patients and 23.75 $\%$ were from out-patient. Of the $61 P$. aeruginosa isolates from in-patients, $62.30 \%$ were from the wards, while $37.70 \%$ were from ICUs .The overall resistance pattern of 80 isolates of P.aeruginosa against each of the FQs (Ciprofloxacin, Ofloxacin, Levofloxacin, Prulifloxacin, Moxifloxacin) is depicted in table no.II. The distribution of each FQ resistance in the in-patients (ICUs and wards) along with out-patients is represented in table no. III.

Inter comparison of overall resistance rates to Ciprofloxacin and Ofloxacin was found significantly higher than those to Levofloxacin and Prulifloxacin $(\mathrm{p}=0.000)$. The overall resistance rates to Moxifloxacin were significantly higher when compared to all other drugs $(\mathrm{p}=0.000)$.A significant resistance $(\mathrm{p}<0.05)$ was observed for Ciprofloxacin, Ofloxacin, and Levofloxacin in the $P$. aeruginosa isolates from the outpatient samples when compared to isolates from ICU samples. Similarly; significant resistance $\quad(p<0.05)$ for Ofloxacin,
Prulifloxacin and Moxifloxacin was observed for $P$. aeruginosa isolates from wards in comparison to the ICUs.

Resistance to antimicrobial agents is an increasing clinical problem and a recognized public health threat. The magnitude of the problem increases several folds when organisms such as $P$. aeruginosa is involved which shows multifaceted resistance mechanisms to the available antibiotics. To tackle the grave situation of antibiotic resistance some of antimicrobial class such as FQs have been obtained by the slight modification of previous generations (Shivani saxena et al., 2014).

FQs are bactericidal and widely used class of antibiotics. Their effectiveness against $P$. aeruginosa is one of their most important features; but prevalence of FQs resistance is related to its intensity of use. Several authors across the globe have reported FQ resistance rate ranging from $12.5 \%$ (Algun et al., 2004) to as high as $73.2 \%$ (Deepak Arora et al., 2011). In the present study the FQs resistance rate ranges from $43.75 \%$ (Moxifloxacin) to $27.5 \%$ (Levofloxacin \& Prulifloxacin).

The overall resistance rate for each of tested $\mathrm{FQs}$ in the present study is as follows. Ciprofloxacin the most active FQ against $P$. aeruginosa was found to be overall resistant in $28.75 \%$ of the isolates. Similar to our finding Kunimoto DY et al., (1999) had reported resistance rate of $30.7 \%$. In various studies investigating the ciprofloxacin resistance in $P$. aeruginosa reported from $0 \%$ to $89 \%$. In current study, ciprofloxacin is resistant in $47.36 \%$ of isolates obtained from out-door patients followed by resistance of $26.31 \%$ in wards and $17.33 \%$ in ICUs. U algun et al., had reported a lower overall resistance rate of $12.5 \%$; while $23.4 \%$ in ICU and $1.9 \%$ in OPD. For Ofloxacin overall $28.75 \%$ resistance was 
observed in this study. Other authors have reported Ofloxacin resistance to be in between $19 \%$ and $62.5 \%$. In the present study, Ofloxacin resistance of $21.7 \%$ in ICUs and $31.57 \%$ in wards was noticed. U algun et al., had reported an overall resistance rate of $19.9 \%$ for Ofloxacin; while $32.8 \%$ in ICU and $7.5 \%$ in OPD.

Overall resistance rate of $27.5 \%$ was noted each for Levofloxacin and Prulifloxacin in this study. Different studies have reported Levofloxacin resistance in between $16.9 \%$ to $36 \%$, while susceptibility reports were higher for Prulifloxacin (72\%) than for Ciprofloxacin $(65 \%)$ and Levofloxacin $(61 \%)$.In the current study, resistance of $21.7 \%$ and $26.08 \%$ was in ICUs alongwith $23.6 \%$ and $28.94 \%$ in wards; for levofloxacin and prulifloxacin respectively. But in out-door patients levofloxacin was resistant in $42.10 \%$ and Prulifloxacin in 26.31\%. U algun et al., had reported a resistance rate of $16.9 \%$; while $29.7 \%$ in ICU and $3.8 \%$ in OPD, for levofloxacin.

In the current study, Moxifloxacin had the highest resistance rate of $43.75 \%$, amongst all the FQs. In the study by Arpana et al., had also reported lower activity of moxifloxacin in gram negative bacteria in comparison to gram positive. This variation in resistance rates of different FQs may be due to the disparity of antibiotic policies with geographical variation, prior monotherapy with FQs, study population and type of sample included in the study.

Table.1 Zone size interpretative table of the FQs used in the study.

\begin{tabular}{|c|c|c|c|c|c|}
\hline \multirow{2}{*}{$\begin{array}{l}\text { Sl. } \\
\text { No }\end{array}$} & \multirow[t]{2}{*}{ Antibiotic } & \multirow{2}{*}{$\begin{array}{c}\text { Drug } \\
\text { content } \\
(\mu \mathrm{gm})\end{array}$} & \multicolumn{3}{|c|}{ Zone Of Inhibition } \\
\hline & & & Resistant & Intermediate & Sensitive \\
\hline 1 & Ciprofloxacin & 5 & $\leq 15$ & $16-20$ & $\geq 21$ \\
\hline 2 & Levofloxacin ${ }^{\#}$ & 5 & $\leq 15$ & $16-18$ & $\geq 19$ \\
\hline 3 & Olfoxacin ${ }^{\#}$ & 5 & 12 & $13-14$ & 15 \\
\hline 4 & Prulifloxacin $^{*}$ & 5 & $\leq 12$ & $13-15$ & $\geq 16$ \\
\hline 5 & Moxifloxacin ${ }^{* * *}$ & 5 & $<17$ & $18-24$ & $\geq 25$ \\
\hline
\end{tabular}

\#The zone diameter is interpreted in accordance to CLSI 2014 guidelines (CLSI antimicrobial susceptibility testing ; vol 34 N0.1;Page :1-230).

*The zone diameter is interpreted for Prulifloxacin is as per Eiken chemical, Japan.

**Zone diameter is interpreted for moxifloxacin as per BSAC recommendation.

Table.2 Overall resistance to each $\mathrm{FQs}$ against the $\mathrm{P}$. aeruginosa isolates $(\mathrm{n}=80)$

\begin{tabular}{|l|l|}
\hline Fluoroquinolones & Percentage of overall resistance \\
\hline Ciprofloxacin $(5 \mu \mathrm{gm})$ & $28.75 \%(23)$ \\
\hline Ofloxacin $(5 \mu \mathrm{gm})$ & $28.75 \%(23)$ \\
\hline Levofloxacin $(5 \mu \mathrm{gm})$ & $27.5 \%(22)$ \\
\hline Prulifloxacin $(5 \mu \mathrm{gm})$ & $27.5 \% \quad(22)$ \\
\hline Moxifloxacin $(5 \mu \mathrm{gm})$ & $43.75 \%(35)$ \\
\hline
\end{tabular}


Table.3 Distribution of resistance pattern for the various FQs among in-patients (ICUs \& wards) and out-patients

\begin{tabular}{|l|l|l|l|l|}
\hline Fluoroquinolones & $\begin{array}{c}\text { Overall resistance in } \\
\text { IPD (ICUs + wards; } \\
\mathrm{n=61)}\end{array}$ & $\begin{array}{c}\text { Resistance in } \\
\text { wards (n=38) }\end{array}$ & $\begin{array}{c}\text { Resistance in } \\
\text { ICU(n=23) }\end{array}$ & $\begin{array}{c}\text { Resistance in } \\
\text { OPD(n=19) }\end{array}$ \\
\hline Ciprofloxacin & $22.95 \%(14)$ & $26.31 \%(10)$ & $17.33 \%(04)$ & $47.36 \%(09)$ \\
\hline Ofloxacin & $27.86 \%(17)$ & $31.57 \%(12)$ & $21.7 \%(05)$ & $31.57 \%(06)$ \\
\hline Levofloxacin & $22.95 \%(14)$ & $23.6 \%(09)$ & $21.7 \%(05)$ & $42.10 \%(08)$ \\
\hline Prulifloxacin & $27.86 \%(17)$ & $28.94 \%(11)$ & $26.08 \%(06)$ & $26.31 \%(05)$ \\
\hline Moxifloxacin & $45.90 \%(28)$ & $52.63 \%(20)$ & $34.78 \%(08)$ & $36.84 \%(07)$ \\
\hline
\end{tabular}

The present study has surprisingly shown that the percentage of FQs resistance in samples obtained from ICU patients was significantly lower than the patients from the OPD and Wards.The only plausible explanation to this may be had from the possible rampant and irrational use of FQs antibiotics in population. Various studies have documented that the single most important determinant of FQ resistance is prior exposure to these drugs. Because of the increasing FQs resistance empirical usage should either abandoned or restricted in order to take the developing resistance rates under control. Also a detailed history of antibiotic usage with special emphasis on prior FQ consumption might give a clue to the origin of resistance in our OPD patients. At the same time, the relative lower rates of resistance in ICU patient, points towards a scientific antibiotic use practice and meticulous attention to the prevention of nosocomial infection.

The limitations of the present study were small sample size and due to financial constraint we did not use $\mathrm{E}$ strips for the determination of MIC values of FQs.A follow up study therefore needs to be conducted which incorporate determination of MIC with appropriate sample size along with details of prior antibiotic use in the study design.The findings also warrant further probe into possible genetic factors in the microbe that may contribute to FQs resistance.

To conclude, although in the present study we observed moderate resistance to FQs, but recent years have witnessed an emergence in FQs resistance. To overcome the antibiotic resistance we need a combined approach consisting of rational use of FQs, avoidance of FQs as monotherapy, improved hospital infection control measures, along with continuous monitoring of local FQs resistance pattern with an aim that the results should be made readily available to the clinician so as to maximize the possibility of administrating appropriate effective antibiotic for better patient management. Thus selection of appropriate antibiotics and its use on the pharmacodynamics concept remains the best way in treating $P$. aeruginosa infections; otherwise we may be nearing to an end of antibiotics era.

\section{Acknowledgement}

Authors acknowledge the immense help received from the scholars whose articles are 
cited and included in references of this manuscript. The authors are also grateful to authors/editors/publishers of all those articles, journals and books from where the literature for this article has been reviewed and discussed.

\section{References}

Axel Dalhoff; Global Fluoroquinolone Resistance Epidemiology and Implictions for Clinical Use" Interdisciplinary Perspectives on Infectious Diseases; Volume 2012; Page: 1-37

Brett H. Heintz andJenanaHalilovic; "Lessons Learned from Surveillance of Antimicrobial Susceptibilities of Pseudomonas aeruginosa at a Large Academic Medical Center ";Pharmaceuticals 2010, Vol: 3, page: 1070-1083

CLSI. 2014. Performance standards for antimicrobial susceptibility testing; twenty-second informational supplement. CLSI document M100S22. Wayne, PA. Clinical and Laboratory Standards Institute.

Deepak arora, Neerja jindal, Rajiv kumar, Romit; "Emerging antibiotic resistance in pseudomonas :a challenge" ; International journal of pharmacy and pharmaceutical sciences issn- 0975-1491 vol 3, issue 2, 2011

Hideki Kobayashi, Miho Isozaki, Tatsuya Fukuda, YojiroAnzai, Fumio Kato; "Surveillance of FluoroquinoloneResistant Clinical Isolates of Pseudomonas aeruginosa"; Open Journal of Medical Microbiology, Vol: 2013, Issue: 3, Page: 144-150

J. M. Andrews, J. P. Ashby, G. M. Jevons, R. Wise "Tentative minimum inhibitory concentration and zone diameter breakpoints for moxifloxacin using BSAC criteria"; Journal of antimicrobial chemotherapy 1999, vol.44, page: 819-822

Kunimoto DY, Sharma S, Garg P, et al.,. In vitro susceptibility of bacterial keratitis pathogens to ciprofloxacin. Emerging resistance. Ophthalmology 1999; vol. 106: page: 80-85

Liam S. Redgrave, Sam B. Sutton, Mark A. Webber, and Laura J.V. Piddock; Fluoroquinolone resistance: mechanisms, impact on bacteria, and role in evolutionary success"; Trends in Microbiology; August 2014; Vol. 22; No. 8; Page: 436-445

Luna Adhikari, K Roy, Dechen C Tsering, Ranabir Pal, SumitKar; "Susceptibility Rates of Pseudomonas Aeruginosa Strains to Quinolones"; Journal of Laboratory Physicians ; Jul-Dec 2010 ; Vol-2 ;Issue-2

Monica Cheesbrough; "District Laboratory Practice in Tropical Countries"; Second Edition; Part-2; Page: 1-434

Simona Roveta, Anna Maria Schito, Anna Marchese, Gian Carlo Schito "Microbiological rationale for the utilisation of prulifloxacin, a new fluoroquinolone, in the eradication of serious infections caused by Pseudomonas aeruginosa" International journal of antimicrobial agents 2005; vol.26; Page 366-372

ShivaniSaxena, Gopa Banerjee, Rajiv Garg, Mastan Singh, S.K.Verma and R.A.S.Kushwaha; "The antibacterial efficacy of different antipseudomonal agents against Pseudomonas aeruginosa" International Journal of Current Microbiology and Applied Sciences; vol: (2014); Issue 3(11); Page: 572-575

Tanya Strateva, Daniel Yordanov;
"Pseudomonas aeruginosa $-\mathrm{a}$
phenomenon of bacterial resistance";


Journal of Medical Microbiology Vol: (2009), Issue 58, Page: 11331148

Topkaya A, Kucukercan M, Oguzoglu N. Inducible beta-lactamaseproduction and susceptibility rates of Pseudomonas aeruginosa strains toaminoglycosides and quinolons. $\mathrm{X}$. Turkish Microbiology and InfectiousDisease Congress Book; 2001.

U Algun, A Arisoy, $\mathrm{T}$ Gunduz, B Ozbakkaloglu; "The resistance of pseudomonas aeruginosa strains to fluoroquinolone group of antibiotics"; Indian Journal of Medical Microbiology; Year : 2004 ; Volume : 22 ; Issue : 2 ; Page : $112-114$ White, R.L.; Friedrich, L.V.; Mihm, L.B.; Bosso, J.A. Assessment of the relationship between antimicrobial usage and susceptibility: differences between the hospital and specific patient-care areas. Clin. Infect. Dis. 2000, 31, 16-23.

\section{How to cite this article:}

Sandhya Mishra, Mrityunjay Kumar, Anamika Vyas and A.S.Dalal. 2016. Antibiogram of Clinical Isolates of Pseudomonas aeruginosa against Selected Quinolones. Int.J.Curr.Microbiol.App.Sci. 5(6): 771-777. doi: http://dx.doi.org/10.20546/ijcmas.2016.506.085 